\title{
Factors influencing public participation in biobanking
}

\author{
Mamoun Ahram ${ }^{\star, 1}$, Areej Othman ${ }^{2}$, Manal Shahrouri ${ }^{3}$ and Ebtihal Mustafa ${ }^{1}$ \\ A diversity of public participation in biobanking is a highlight for the success of biobanks. It was previously reported that \\ only two-thirds of Jordanians expressed interest in biobanking. To promote public involvement in a biobank, it is imperative \\ to determine the aspects that influence the decision-making to participate. On the basis of a national survey involving 3196 \\ respondents, the influence of 13 biobanking factors was assessed, including returning research results, privacy, freedom of \\ choice, uncertainties about research, monetary and health considerations, and personal belief. Perception toward each factor \\ was also correlated with willingness to participate in a biobank. A considerable number of respondents indicated returning \\ research results as influential in their decision to become biobank donors. Interestingly, whereas the positive perception of \\ availability of general results (39\%) correlated with willingness to donate for biobanking, the negative view of unavailability \\ of individualized results $(47 \%)$ did not correlate with unwillingness. Religious permission of sample donation for research had \\ the strongest positive influence $(61 \%)$ and the highest correlation to participate among positively perceived factors. Unspecified \\ research was highly indicated as a negative factor (45\%), but did not correlate with unwillingness to become a biobank donor, \\ whereas allowed withdrawal had a positive effect (31\%) and correlation to contribute to biobanking. The negative perception \\ of accessing medical information (9.5\%) and re-contact $(8.5 \%)$ had the strongest correlation with unwillingness to donate \\ to a biobank. These results may provide an insight into how to formulate strategies to promote public participation in \\ biomedical research and biobanking.
} European Journal of Human Genetics (2014) 22, 445-451; doi:10.1038/ejhg.2013.174; published online 7 August 2013

Keywords: biobanking; research participation; religion; returning results; benefit; privacy

\section{INTRODUCTION}

The growing number of biobanks worldwide is a reflection of their potential in increasing the reproducibility and meaningfulness of data generated from biomedical research. Reproducibility stems from the fact that biospecimens are collected by strict and standardized methodologies, while meaningfulness is a result of linking relevant information to the biospecimens themselves as well as their donors. The success of biobanks depends on the participation of diverse groups of individuals. Such diversity leads to understand the molecular causes of diseases and unraveling their differential distribution among the various ethnic groups and populations. Nevertheless, biobanks are associated with a number of ethical, social, and legal components. Some of the controversial themes that involve the donors include the existence and type of consent form, the right of withdrawal, personal benefit, returning research results, and protection of privacy. Arguments also surround the samples such as the nature of research, participating researchers, and storage period of biospecimens and information.

Jordan is a nation strategically located in the central region of the Middle East. It has developing biomedical research activities and is considered as a favorite destination for health care in the region due to its advanced medical system and renowned physicians. The country hosts a heterogeneous mixture of different and unique ethnic groups. Hence, the existence of national or disease-specific biobanks would foster understanding the molecular diversity of the Jordanian population and establish a goal-oriented biomedical research. On the basis of a national survey, we have previously reported that $64 \%$ of the Jordanian public expressed willingness to participate in biobanking via the donation of biospecimens and relevant information. ${ }^{1}$ However, a considerable one-third of respondents did not desire to contribute toward biobanking. A small-scale study involving hospital visitors in Saudi Arabia, a neighboring country, revealed a similar rate of enthusiasm toward biobanking. ${ }^{2}$ Although these rates are similar to or better than ratings obtained in Southern and Eastern European nations, ${ }^{3}$ they are lower than those obtained in Northern European countries. ${ }^{4-6}$ Variations of attitudes toward biobanking and research also exist among different ethnic groups within the same country. ${ }^{7-10}$ Hoeyer $^{11}$ emphasized the consideration of these attitudinal variations when establishing a biobank as it illustrates respect toward the various groups of society.

The rate of willingness to participate in biobanking among the public in Jordan does not reflect the high level of enthusiasm toward biomedical research. In fact, $98 \%$ of respondents of the national survey agreed to the concept of funding biomedical research at the national level. ${ }^{12}$ These seemingly conflicting results necessitate deeper understanding of the factors that influence public decision for biobank participation. Once influential factors are identified, it becomes easier to plan for educational programs to increase the awareness toward biomedical research and, particularly, biobanking. As part of the aforementioned national survey, the influence of 13 factors was investigated. The factors could be divided into six categories, namely, monetary or health considerations, privacy,

${ }^{1}$ Department of Physiology and Biochemistry, Faculty of Medicine, Amman, Jordan; ${ }^{2}$ Department of Maternal Child Health Nursing, Faculty of Nursing, The University of Jordan, Amman, Jordan; ${ }^{3}$ Office of Science, Research, and Health Policy, King Hussein Institute for Biotechnology and Cancer, Amman, Jordan

${ }^{*}$ Correspondence: $\operatorname{Dr} M$ Ahram, Department of Physiology and Biochemistry, Faculty of Medicine, Amman 11942, Jordan. Tel: +962 $65355000 / 23481$; Fax: + 9626530 0820; E-mail: dr.ahram@gmail.com

Received 4 March 2013; revised 27 June 2013; accepted 2 July 2013; published online 7 August 2013 
freedom of choice of donors, availability of research results, uncertainties related to research projects, and personal belief. These factors were selected as being among the most controversial issues in biobanking and/or presumed to have implications on the decisionmaking of the Jordanian public. ${ }^{13,14}$ Due to the similar cultural background between Jordan and surrounding countries, the data generated and discussed herein may provide means of understanding and accessing the public in the region.

\section{MATERIALS AND METHODS}

\section{The survey}

Questions related to biobanking were part of a nation-wide, structured, cross-sectional survey entitled 'Knowledge, Attitudes, Practices towards Cancer Prevention and Care in Jordan' as previously described. ${ }^{1,12,15}$ The survey included in-depth interviews with a community-based sample during the period of January-March 2011. The questionnaire was designed and developed by a panel of research experts from clinical and academic institutions taking into consideration the local ethical and cultural context.

The sample was composed of 3196 individuals aged 18 years and above representing the Jordanian population. The sampling design was carried out in collaboration with the Department of Statistics (DOS) in Jordan. The sampling frame was based on the 2004 census and designed to provide estimates for the 12 governorates of Jordan. According to DOS sampling guidelines, the minimum sample size representing the Jordanian population was reached regardless of non-response rates. Interviews were conducted face-to-face in the interviewees' households by trained field workers. Random selection of individuals interviewed from each household was achieved using the Kish table procedure. Repeated visits were attempted to obtain responses of eligible respondents. No demographic data were collected from those who were not available at the time of interview and those who refused to participate.

The demographics analyzed in association with responses included: gender, age, education, income, and marital status (Supplementary Table 1).

\section{Components of the questionnaire}

The concepts of biospecimens and biobanking were introduced to survey respondents. The influence of a number of statements regarding decision to participate in biobanking was then assessed by asking survey participants the following question: 'Will your decision to donate biospecimens and information [for biobanking] be influenced positively or negatively, or will it have no effect if you know that (statement)?' (Table 1). The statements covered different aspects of biobanking and could be divided into the following six categories: monetary or health considerations, privacy of donors, freedom of choice, availability of research results, uncertainties related to research projects, and personal belief. Respondents would then rate each statement as positive, negative, no effect, or do not know; 'positive' indicates that the statement would motivate individuals to participate in the biobank; 'negative' indicates that it would discourage individuals from participating; and 'no effect' implies neutral position on decision to participate.

Participants were then asked about their willingness to donate biological samples as well as personal information and information related to their health and family. Responses to the last question were measured on a 5-point Likert scale with scores ranging from 'very likely' to 'very unlikely', in addition to a category of 'do not know.'

\section{Data analysis}

Data were analyzed using the SPSS software program, version 17.0 (SPSS Inc. Chicago, IL, USA). Descriptive statistics were used to report sample characteristics in addition to frequencies and percentages. Pearson Chi-square was used to assess the relationship between individual responses within each statement with demographics. The correlations between responses within each statement and the overall willingness to participate in biobanking were assessed by calculating phi-coefficient. Before this analysis, the two categories of likelihood to participate in biobanking were grouped together as well as the two groups of unlikelihood, whereas the 'do not know' category was excluded from analysis due to its small size $(0.34 \%)$ yielding a final sample size of 3185 . Detailed data and statistical analyses are provided in Supplementary File.

\section{RESULTS}

\section{Overview of the data}

The overall distribution of perception of the 13 statements on the decision to participate in biobanking is presented in Figure 1. Upon ranking statements based on their influence (Table 2), the three most positively influential statements were religious permission of donating biospecimens for the purpose of scientific research (61.2\%), followed by availability of general results (39.2\%), and allowed withdrawal (31.1\%) (Table 2A). On the other hand, the aspects of biobanking that had the highest negative influence were unavailability of personalized results to the donor (46.9\%), involvement of samples in non-specified research $(44.8 \%)$, and indefinite storage of samples (21.2\%) (Table 2B). The three highest non-influential statements were all related to monetary and health considerations with lack of monetary benefit to donors ranking first (76.9\%), followed by lack of health benefit $(72.2 \%)$, and lack of monetary cost $(69.4 \%)$ (Table 2C). Only two statements had more 'positive' or 'negative' effect than 'no effect' on the decision to participate in biobanking. The first was religious permission $(61.2 \%$ positive $v s 35.5 \%$ no effect)

Table 1 Will your decision to donate biological sample (s) and information (for biobanking) be influenced positively, negatively, or it will have no effect if you know that 'statement'?

\begin{tabular}{|c|c|c|}
\hline Criteria & Label & Statement \\
\hline \multirow[t]{2}{*}{ Return of research results } & 1. Unavailability of personalized results & Donors will not be provided with results related to their samples \\
\hline & 2. Availability of general results & Donors will be provided with research results in general \\
\hline \multirow[t]{3}{*}{ Uncertainties about research } & 3. Non-specified research & The specific research study is not specified \\
\hline & 4. Participation of international researchers & Researchers from outside of Jordan can participate \\
\hline & 5. Indefinite storage of samples & Samples will be stored indefinitely \\
\hline \multirow[t]{3}{*}{ Monetary and health consideration } & 6. Lack of monetary benefit & Donors will not be paid for donation \\
\hline & 7. Lack of health benefits & There is no direct health benefit to donors \\
\hline & 8. Lack of monetary cost & Donation of samples will not monetary cost donors \\
\hline \multirow[t]{2}{*}{ Privacy } & 9. Re-contact & Donors can be contacted to update donor's information \\
\hline & 10. Access to medical file & Health information can be obtained from donor's physician or medical file, if necessary \\
\hline \multirow[t]{2}{*}{ Freedom of choice } & 11. Allowed withdrawal & Donors can withdraw at anytime without any repercussion \\
\hline & 12. Existence of signed consent & Consent of donors will be obtained before donation \\
\hline Personal belief & 13. Religious permission & Religion does not prohibit individuals from donating samples \\
\hline
\end{tabular}




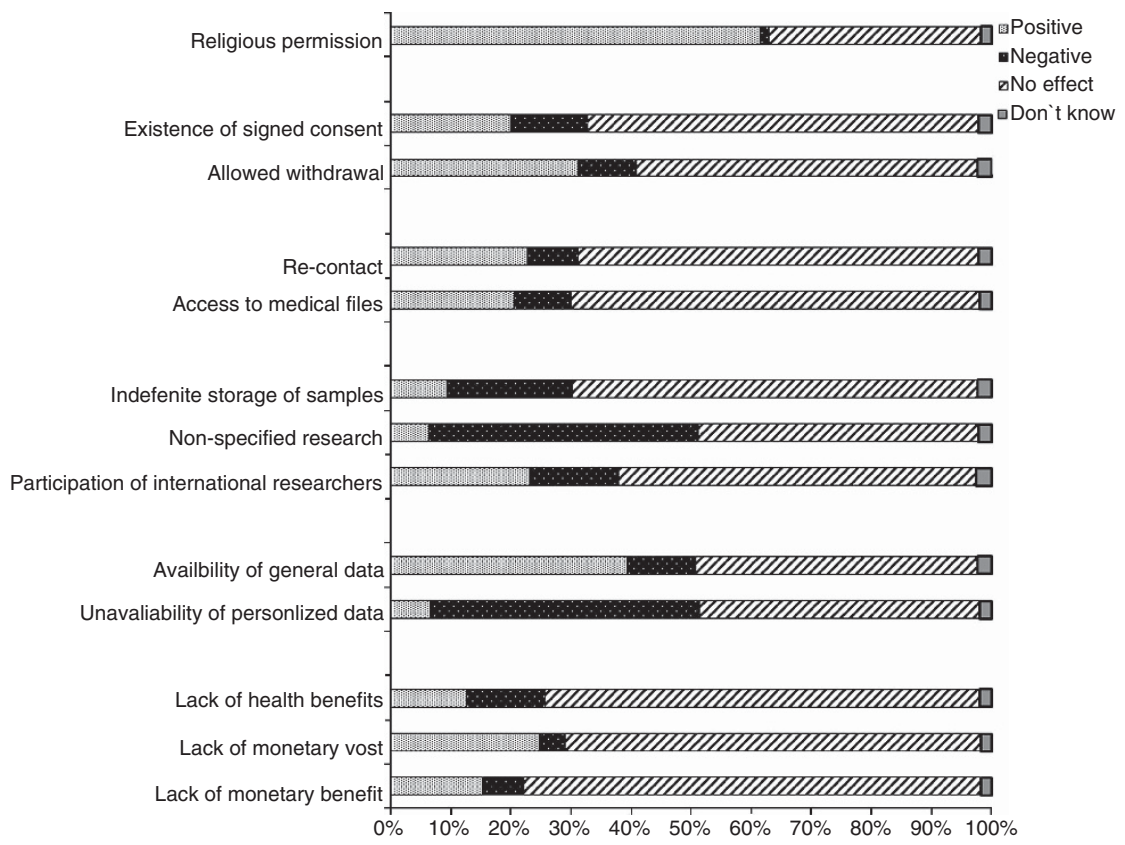

Figure 1 The influence of 13 factors on public decision to participate in biobanking. Factors may have positive, negative, or no influence. In addition, a 'do not know' option is also provided.

Table 2 Ranking of factors influencing decision to participate in biobanking according to degree of influence ${ }^{a}$

\begin{tabular}{|c|c|c|c|c|c|}
\hline \multicolumn{2}{|l|}{ A. Positive influence } & \multicolumn{2}{|l|}{ B. Negative influence } & \multicolumn{2}{|l|}{ C. No influence } \\
\hline & Responses, & & Responses, & & Responses, \\
\hline Label & $\%^{\mathrm{a}}$ & Label & $\%^{\mathrm{a}}$ & Label & $\%^{\mathrm{a}}$ \\
\hline 1. Religious permission & 61.2 & 1. Unavailability of personalized results & 46.9 & 1. Lack of monetary benefit & 76.2 \\
\hline 2. Availability of general results & 39.2 & 2. Non-specified research & 44.8 & 2. Lack of health benefit & 72.6 \\
\hline 3. Allowed withdrawal & 31.1 & 3. Indefinite storage of samples & 21.2 & 3. Lack of monetary cost & 69.4 \\
\hline 4. Lack of monetary cost & 24.6 & 4. Participation of international researchers & 14.8 & 4. Access to medical file & 68.1 \\
\hline $\begin{array}{l}\text { 5. Participation of international } \\
\text { researchers }\end{array}$ & 23.0 & 5. Existence of signed consent & 13.0 & 5. Indefinite storage of samples & 67.5 \\
\hline 6. Re-contact & 22.7 & 6. Lack of health benefit & 13.0 & 6. Re-contact & 66.7 \\
\hline 7. Access to medical file & 20.4 & 7. Availability of general results & 11.5 & 7. Existence of signed consent & 65.0 \\
\hline 8. Existence of signed consent & 19.8 & 8. Allowed withdrawal & 9.8 & 8. Participation of international researchers & 59.6 \\
\hline 9. Lack of monetary benefit & 15.2 & 9. Access to medical file & 9.5 & 9. Allowed withdrawal & 56.8 \\
\hline 10. Lack of health benefits & 12.5 & 10. Re-contact & 8.5 & 10. Availability of general results & 47.0 \\
\hline 11. Indefinite storage of samples & 9.1 & 11. Lack of monetary benefit & 6.8 & 11. Non-specified research & 46.9 \\
\hline $\begin{array}{l}\text { 12. Unavailability of personalized } \\
\text { results }\end{array}$ & 6.8 & 12. Lack of monetary cost & 4.4 & 12. Unavailability of personalized results & 44.2 \\
\hline 13. Non-specified research & 6.2 & 13. Religious permission & 1.5 & 13. Religious permission & 35.5 \\
\hline
\end{tabular}

a Due to the small size of the 'do not know' responses to the influence of each statement on their decision to participate in biobanking, they are not shown and can be found in the Supplementary file.

and the second factor was the unavailability of personalized data (46.9\% negative vs $44.2 \%$ no effect).

\section{Returning research results}

Respondents appeared eager to learn individualized research results where $47 \%$ of respondents thought that not having such results would have a negative influence on their decision to participate in biobanking (Table 2B). In contrast, $\sim 44 \%$ did not think it affected their decision. Increasing education was associated with the negative perception of not receiving personalized results $(P=0.002)$ (Supplementary Table 2).

The former negative perception was offset by the possibility of receiving generalized results where $39.2 \%$ thought of it as having a positive influence on their decision to become biobank donors, in contrast to $47 \%$ being not affected (Table 2). There is a significant association in response to receiving generalized results with gender where females were more likely positively influenced than males $(P=0.005)$ who were significantly more indifferent $(P<0.001)$ (Supplementary Table 3 ). A positive perception of receiving general results was also associated with those of higher education $(P<0.001)$ and medium income $(P<0.001)$.

\section{Uncertainties related to research}

The inability to know the type of research had the second highest negative impact among all statements where almost $45 \%$ viewed it negatively (Table 2B). This negative attitude was significantly associated with being female $(P=0.004)$, increasing education $(P<0.001)$, and having medium income $(P=0.032)$ (Supplementary Table 4). It was encouraging, though, to note that more respondents $(23 \%)$ positively viewed the participation of 
international researchers than those who perceived it as a negative aspect (14.8\%) (Figure 1; Table 2). The positive view was associated with increasing education $(P<0.001)$ and having medium income $(P<0.001)$ (Supplementary Table 5$)$. Considerably, individuals aged 60 years old and above had a more negative perception $(P<0.001)$ than a positive one $(P=0.019)$. A negative view of the participation of international researchers was also associated with decreasing education $(P=0.002)$.

More respondents $(21.1 \%)$ were not in favor of having their samples stored for an unspecified period of time than those who thought otherwise $(9.1 \%)$ (Table $2 \mathrm{~A}$ and $\mathrm{B}$, respectively). However, the decision to participate in biobanking of almost two-thirds of respondents $(67.5 \%)$ would not be affected (Table $2 \mathrm{C})$. Whereas the decision of males was slightly not affected by this procedure of a biobank $(P=0.048)$, females were significantly more positive $(P=0.002)$ (Supplementary Table 6$)$. Individuals with lower income were less positive than respondents with medium and high income $(P=0.006)$. On the other hand, individuals with higher income were more positive and less influenced than the other two groups $(P<0.001)$. Lower education was also significantly associated with being most negative $(P=0.020)$, least positive $(P=0.024)$, and least affected $(P=0.034)$ by the indefinite storage of samples.

\section{Monetary and health considerations}

Almost three quarters of respondents did not think that lack of health or monetary benefit in return to their contribution to the biobank would affect their decision to participate (Figure 1; Table 2C). The no-influence to the absence of both benefits was associated with higher income $(P<0.001)$ (Supplementary Tables 7 and 8). A similar association with income was found among the $72.6 \%$ of respondents who were not influenced by the lack of monetary cost when donating for biobanking (Supplementary Table 9). It was intriguing to note that twice more respondent thought that the lack of monetary compensation for donation was positive than those who considered it as negative $(15.2 \%$ vs $6.8 \%$, Table $2 \mathrm{~A}$ and $\mathrm{B}$, respectively). In addition, the lack of monetary cost also had a five-time more positive impact than those who viewed it as negative (24.6 vs 4.4\%; Table $2 \mathrm{~A}$ and $\mathrm{B}$, respectively). An equal proportion of respondents ( $\sim 13 \%)$ rated not having health benefit as either positive or negative (Table $2 \mathrm{~A}$ and $\mathrm{B}$ ). A positive influence for the lack of monetary benefit was significantly more associated with females $(P=0.017)$ and those with lower and medium income $(P=0.02)$. In addition, individuals aged 60 years and above had a significant association with the negative view of lack of monetary cost $(P<0.001)$, monetary benefit $(P<0.001)$, and health benefit $(P=0.008)$.

\section{Privacy}

Approximately two-thirds of respondents did not seem to be influenced about updating information directly by contacting them or indirectly through accessing their medical files or physicians (Table 2C). In addition, a higher rate of a positive attitude than a negative connotation was indicated toward re-contact (22.8 vs 8.5) and access of medical information (20.4 vs 9.5) (Table 2A and B). As for the possibility of re-contacting donors, a significant positive influence was found among those with higher education $(P=0.04)$ and middle income $(P=0.006)$, whereas a negative influence was significantly associated with individuals aged 60 years and above followed by those within the $30-39$ age group $(P<0.001)$ and individuals with higher education $(P<0.04) \quad$ (Supplementary Table 10). The lack of influence of re-contact was associated with higher income $(P=0.03)$ and among those in the age groups of
40-49 and 50-59 $(P=0.019)$. Individuals with higher income were more indifferent and less positive about accessing medical information $(P<0.001)$, but individuals with lower income were less negative $(P<0.001)$ (Supplementary Table 11$)$. Males $(P=0.042)$ and those with higher education $(P=0.004)$ were also more negative.

\section{Freedom of choice}

Although more than half of respondents did not think that having the choice to withdraw would affect their decision to donate for a biobank (Table 2C), a substantial one-third of them $(31.2 \%)$ thought it was a positive aspect. Those with lower education were less positive $(P<0.001)$ (Supplementary Table 12). In addition, individuals aged 60 years and above were less positive and more negative $(P<0.001)$. Respondents within the different categories of income had significantly variable attitudes in which the lower income group was least positive $(P<0.001)$, those with higher income were least negative $(P<0.001)$, and middle income respondents were least influenced by the option of withdrawal $(P=0.002)$.

The presence of a signed consent did not have a significant impact on the respondents' decision to participate (Table 2C). Approximately $20 \%$ of respondents identified obtaining a consent would have a positive influence on their decision to donate (Table $2 \mathrm{~A}$ ), whereas $13.1 \%$ rated it as having a negative connotation (Table $2 \mathrm{~B}$ ). Statistical significance was found between negative perception of consent and being 60 years old and above $(P=0.006)$ (Supplementary Table 13). Association between those who had medium income with positive perception of a signed consent was also significant $(P=0.003)$.

\section{Personal belief}

As religious institutions have a social authority within the Jordanian society, we assessed the influence of the statement that religion does not prohibit individuals from donating biospecimens for research and biobanking. More than $60 \%$ of respondents thought that religious permission of biospecimens donation for research had a positive influence; in contrast to $35.5 \%$ of respondents who were neutral (Figure 1). The positive view of the role of religion was significantly associated with increasing education $(P<0.001)$, whereas decreasing educational status was associated with being least affected $(P=0.016)$ (Supplementary Table 14). Furthermore, individuals with medium income were more likely positively influenced by this statement followed by those within the lowest income category $(P<0.001)$. In contrast, respondents with highest income were least affected $(P<0.001)$.

\section{Summary of responses in relation to demographics}

Three demographic factors have disparate trends in the decisionmaking process to participate in biobanking than others: income, education, and age. Individuals in the highest income category were more likely unaffected by many factors, namely: the lack of payment, cost, and health benefit for participation, re-contact, access to medical information, indefinite storage of samples, existence of a signed consent, and religious permission. In addition, there was a significant correlation between increasing income and likeliness to donate biospecimens for biobanking $(r=0.075, P<0.001$; Supplementary Table 15). Increasing willingness to donate for biobanking was also correlated with increasing education $(\mathrm{r}=0.097, \quad P<0.001$; Supplementary Table 15). Individuals with higher education had a more positive view of the top factors that would motivate participation in a biobank. The factors included religious permission, availability of general research results, allowed withdrawal, participation of international researchers, and re-contact. In contrast, older 
Table 3 Ranking of factors influencing decision to participate in biobanking according to correlation with willingness to donate biospecimens for biobanking

\begin{tabular}{|c|c|c|c|c|c|}
\hline \multicolumn{2}{|c|}{ A. Positive influence } & \multicolumn{2}{|c|}{ B. Negative influence } & \multicolumn{2}{|c|}{ C. No influence } \\
\hline Label & $\phi$-Coefficient (P) & Label & $\phi$-Coefficient (P) & Label & $\phi$-Coefficient (P) \\
\hline 1. Religious permission & $0.162(<0.001)^{*}$ & 1. Re-contact & $-0.185(<0.001)^{*}$ & 1. Lack of health benefit & $0.119(<0.001)^{*}$ \\
\hline 2. Availability of general results & $0.150(<0.001)^{*}$ & 2. Access to medical file & $-0.160(<0.001)^{*}$ & 2. Lack of monetary cost & $0.119(<0.001)^{*}$ \\
\hline 3. Allowed withdrawal & $0.129(<0.001)^{*}$ & 3. Lack of monetary benefit & $-0.153(<0.001)^{*}$ & 3. Indefinite storage of samples & $0.113(<0.001)^{*}$ \\
\hline $\begin{array}{l}\text { 4. Participation of international } \\
\text { researchers }\end{array}$ & $0.081(<0.001)^{*}$ & 4. Allowed withdrawal & $-0.137(<0.001)^{*}$ & 4. Lack of monetary benefit & $0.112(<0.001)^{*}$ \\
\hline 5. Existence of signed consent & $0.079(<0.001)^{*}$ & 5. Lack of monetary cost & $-0.128(<0.001)^{*}$ & 5. Re-contact & $0.101(<0.001)^{*}$ \\
\hline $\begin{array}{l}\text { 6. Unavailability of personalized } \\
\text { results }\end{array}$ & $0.069(<0.001)^{*}$ & 6. Availability of general results & $-0.125(<0.001)^{*}$ & 6. Access to medical file & $0.094(<0.001)^{*}$ \\
\hline $\begin{array}{l}\text { 7. Access to medical file } \\
\text { 8. Re-contact }\end{array}$ & $\begin{array}{l}0.057(0.001)^{*} \\
0.054(0.002)^{*}\end{array}$ & $\begin{array}{l}\text { 7. Existence of signed consent } \\
\text { 8. Indefinite storage of samples }\end{array}$ & $\begin{array}{l}-0.118(<0.001)^{*} \\
-0.115(<0.001)\end{array}$ & $\begin{array}{l}\text { 7. Existence of signed consent } \\
\text { 8. Participation of international }\end{array}$ & $\begin{array}{l}0.057(0.001)^{*} \\
0.032(0.070)\end{array}$ \\
\hline 9. Non-specified research & $0.047(0.008)^{*}$ & 9. Lack of health benefit & $-0.110(<0.001)^{*}$ & $\begin{array}{l}\text { researchers } \\
\text { 9. Non-specified research }\end{array}$ & $0.008(0.647)$ \\
\hline 10. Lack of monetary cost & $0.038(0.030)^{*}$ & $\begin{array}{l}\text { 10. Participation of international } \\
\text { researchers }\end{array}$ & $-0.088(<0.001)^{*}$ & 10. Allowed withdrawal & $0.000(0.997)$ \\
\hline 11. Indefinite storage of samples & $0.030(0.087)$ & 11. Religious permission & $-0.063(<0.001)^{*}$ & $\begin{array}{l}\text { 11. Unavailability of personalized } \\
\text { results }\end{array}$ & $-0.009(0.606)$ \\
\hline $\begin{array}{l}\text { 12. Lack of monetary benefit } \\
\text { 13. Lack of health benefits }\end{array}$ & $\begin{array}{l}0.027(0.128) \\
0.010(0.583)\end{array}$ & $\begin{array}{l}\text { 12. Non-specified research } \\
\text { 13. Unavailability of personalized } \\
\text { results }\end{array}$ & $\begin{array}{l}0.002(0.889) \\
0.016(0.356)\end{array}$ & $\begin{array}{l}\text { 12. Availability of general results } \\
\text { 13. Religious permission }\end{array}$ & $\begin{array}{l}-0.032(0.075) \\
-0.111(<0.001)^{*}\end{array}$ \\
\hline
\end{tabular}

*Significant $(P<0.05)$.

individuals (60 years old and above) had a more negative view of biobanking (Supplementary Table 15), in general, and the factors that are related to participation of international researchers, the lack of payment, cost, and health benefit, existence of a signed consent, allowed withdrawal, re-contact, and indefinite storage of samples.

Correlation of factors with willingness to participate in biobanking Approximately, $64 \%$ of respondents expressed their willingness to donate biospecimens and information for biobanking, whereas almost $34 \%$ were unlikely to donate. ${ }^{1}$ Willingness to participate in biobanking correlated with increasing education, increasing income, decreasing age, and being single or married (Supplementary Table 15). To learn which of the factors was more associated with the potential willingness (or unwillingness) to donate for biobanking, we correlated the likeliness of participation with the positive (Table 3A), negative (Table 3B), and lack of influence (Table 3C) using phi-coefficient analysis (detailed data are presented in Supplementary Table 16). The positive influence of religious permission had the strongest correlation with the willingness to participate in biobanking among factors having a positive perception $(\phi=0.162$, $P<0.001$ ). This was followed by the positive influence of availability of general results $(\phi=0.150, P<0.001)$ and allowed withdrawals $(\phi=0.129, P<0.001)$ (Table 3A). Interestingly, the positive perception of participation of international researchers ranked fourth with a $\phi$-value of $0.081(P<0.001)$. Likeliness to participate in biobanking also correlated with the lack of influence of absence of health $(\phi=0.119, P<0.001)$ and monetary benefits $(\phi=0.112, P<0.001)$ ranking first and fourth, in terms of neutral perception toward the statements (Table 3C). In addition, the no-influence of lack of monetary cost correlated positively with intention to participate in biobanking $(\phi=0.119, P<0.001)$ as well the indefinite storage of samples $(\phi=0.113, P<0.001)$.

It is interesting to note that although the unavailability of personalized research results and the involvement of biospecimens in non-specified research had the highest negative perception of biobanking (Table 2B), they were the only negatively viewed statements that did not correlate with unwillingness to participate in biobanking (Table 3B). Rather, the negative view of privacy-related items, namely re-contact and accessing medical information, ranked highest when correlated with unwillingness to donate for biobanking, with $\phi$-values of -0.185 and $-0.150(P<0.001)$, respectively.

\section{DISCUSSION}

This study reveals the existence of two main factors that can promote public participation in biobanking in Jordan: returning research results and religious permission. Returning research results was found to be an excellent incentive to drive public donation for biobanking in our study as well in other European countries and the United States. ${ }^{16,17}$ Although the inability of survey participants to access research results of their samples initially appeared to dissuade people from donating their biospecimens, it did not correlate with the unwillingness to participate in biobanking. These findings are in line with two studies showing that the unavailability of specific research results did not seem to hinder participation in biobanking and can only be for motivational purposes. ${ }^{13,18}$ It is with no doubt that returning research results has many advantages such as showing respect toward participants, educating them, gaining their trust, and increasing their interest in research. ${ }^{19,20}$ However, drawbacks also exist including the need of financial and logistical support, the fear of misreporting data, and the association of research data with negative psychological and social burden without clinical benefit. ${ }^{19-21}$ We suggest the formation of an advisory committee that includes both biobanking administrators and researchers as well as members of the public to decide on the type of research results and best mechanisms of sharing them with the public. The dissemination of generalized research results in the form of periodic newsletters and scientific articles may be a satisfactory solution and a practical proof of the outcome of public contribution toward research. Other modes of communicating research results can be via public forms and biobanking web sites, which allow participants to be more interactive. $^{22}$

A main concern among the Finnish public who declined to consent to DNA biobanking was not knowing for what their samples would be used. ${ }^{5}$ A similar concern was reported among Swedish research participants who refused to biobank their own DNA samples. ${ }^{23}$ In our study, there was a clear concern about not knowing the type of research in which the donated samples would be involved. Nevertheless, such possible operational procedure did not seem to drive respondents away from participating in biobanking activities as it did not correlate with unwillingness to donate for a biobank. In spite of that, assurances can be provided to donors by several means such as the right to withdraw, which was favored by almost one-third of the Jordanian public and correlated significantly with the intention 
to participate in biobanking, and providing future participants different levels of informed consent to choose from. We previously reported that one-fourth of those willing to donate for biobanking studies preferred a consent form restricted by either research type or number of involved studies. ${ }^{12}$ Another level of assurance can be via informing the public of the involvement of research ethics committees, an option favored by a considerable proportion of potential biobanking participants. ${ }^{5-7}$

Jordan is a predominantly Islamic state with a Christian minority ( $\sim 6 \%)$. People belonging to both religions respect their beliefs, venerate religious leaders, and are guided by religious teachings in their daily affairs. Religious approval of sample donation for research appeared to have a positive influence in the decision to participate in biobanking. Opinions of Malay-Muslims in Singapore and UK Muslims toward biospecimens donation and biobanking were shown to be negatively shaped by presumed religious beliefs. ${ }^{9,10}$ The influence of religion on research is also apparent in non-Muslim cultures as well. For example, a pan-European study found an inverse correlation between belief in God and providing information to a biobank. ${ }^{24}$ In addition, the UK Human Genetics Commission ${ }^{25}$ reported a connection between the degree of religious attachment and negative view of genetic research. As Islam has historically been associated with scientific knowledge and advancement, ${ }^{26}$ religious institutions may be utilized to increase the awareness toward research. In fact, a recent awareness campaign organized by a cancer center in Jordan utilized three religious figures among others to promote early cancer detection. The religious factor may not be a determining factor in biobank participation; rather, it is expected to provide comfort into making the decision to enroll in research initiatives.

Although there is a strong correlation between unwillingness to participate in biobanking with the negative perception of the possibility of re-contact and accessing medical information, it is interesting to note the low effect of privacy-related issues. This is apparent from the high rating of the no-influence and the minimal negative effect in response to the possibility of re-contact and accessing medical information. There is even a higher positive view to both criteria than a negative one. Moreover, we reported no difference in donating both biospecimens and information among potential biobank participants. ${ }^{1}$ In a recent genetic study involving over 250 patients of multiple sclerosis, there was unanimous approval among participants to be re-contacted or have their information updated (Ahram et al, submitted). This is contrary to what has been reported in other cultures where a high level of concern about protection of privacy was measured. ${ }^{5,13,15,27,28}$ Our observations may reflect high level of trust toward researchers in the scientific and medical profession in Jordan and/or a high level of interest in research. ${ }^{12}$ As far as re-contact is concerned, we believe that a balance must be established between informing biobank participants and its mechanism and frequency. It can be left to the biobank facility itself according to its capability and recommendations of an advisory committee that include members of the public.

The presented data add support for biobanking establishment in Jordan. A significant strength of our study is the involvement of a representative sample of the Jordanian population and the simultaneous examination of multiple factors. However, being a self-report interview study, individuals may be reluctant to explicitly state their views objectively and might rather provide biased, socially acceptable answers. Furthermore, this survey was part of a long questionnaire with possible insufficient time for respondents to think about this new concept and this might have contributed to the 'no effect' phenomenon observed in the responses. This study also assessed factors influencing the future intention to participate in biobanking, which may not reflect actual behavior. In fact, it is worth noting that the Jordanian cancer and multiple sclerosis patients had better actual rate and openness of participation in biobanking than the intended rate of the general public (Al-Husseini, personal communication; Ahram et al, submitted). Our results illustrate a general public agreement to participate in biobanking and emphasize the need to establish awareness campaigns to promote public involvement in biomedical research, which correlate with increased level of participation in biobanking. ${ }^{3,24}$ Understanding the driving or deterring factors can be utilized to promote public participation in research. Biobank administrators and researchers in collaboration with diverse groups of the society can work along these factors to design a competent biobank.

\section{CONFLICT OF INTEREST}

The authors declare no conflict of interest.

\section{ACKNOWLEDGEMENTS}

This work was supported by the Arab Fund for Economic and Social Development (AFESD). The KAP Survey was implemented by King Hussein Institute for Biotechnology and Cancer (KHIBC) under The National Life Science Research and Biotechnology Promotion (LSR/BTP) Initiative in Jordan.

1 Ahram M, Othman A, Shahrouri M: Public perception towards biobanking in Jordan. Biopreserv Biobank 2012; 10: 361-365.

2 Al-Jumah MA, Abolfotouh MA: Public perception and attitude of Saudis toward organ and tissue donation. Biopreserv Biobank 2011; 9: 21-27.

3 Gaskell G, Gottweis H, Starkbaum J et al: Publics and biobanks: Pan-European diversity and the challenge of responsible innovation. Eur J Hum Genet 2013; 21: 14-20.

4 Kettis-Lindblad A, Ring L, Viberth E, Hansson MG: Genetic research and donation of tissue samples to biobanks. What do potential sample donors in the Swedish general public think? Eur J Public Health 2006; 16: 433-440.

5 Tupasela A, Sihvo S, Snell K, Jallinoja P, Aro AR, Hemminki E: Attitudes towards biomedical use of tissue sample collections, consent, and biobanks among Finns. Scand J Public Health 2010; 38: 46-52.

6 Cousins G, McGee H, Ring L et al: Public Perceptions of Biomedical Research a Survey of the General Population in Ireland. Dublin: Health Research Board, 2005.

7 Chen DT, Rosenstein DL, Muthappan P et al: Research with stored biological samples: what do research participants want? Arch Intern Med 2005; 165: 652-655.

8 Kaufman DJ, Murphy-Bollinger J, Scott J, Hudson KL: Public opinion about the importance of privacy in biobank research. Am J Hum Genet 2009; 85: 643-654.

9 Medical Research Council and Wellcome Trust: Public Perceptions of the Collection of Human Biological Samples. London: Medical Research Council, 2001; http://www.ukbiobank.ac.uk/wp-content/uploads/2011/07/Public-Perceptions-Collection-Human-Biological-Samples.pdf (last accessed on 26 June 2013).

10 Wong ML, Chia KS, Wee S et al: Concerns over participation in genetic research among Malay-Muslims, Chinese and Indians in Singapore: a focus group study. Community Genet 2004; 7: 44-54.

11 Hoeyer K: Donors perceptions of consent to and feedback from biobank research: time to acknowledge diversity? Public Health Genomics 2010; 13: 345-352.

12 Ahram M, Othman A, Shahrouri M: Public support and consent preference for biomedical research and biobanking in Jordan. Eur J Hum Genet 2013; 21: 567-570.

13 Beskow LM, Dean E: Informed consent for biorepositories: assessing prospective participants' understanding and opinions. Cancer Epidemiol Biomarkers Prev 2008, 17: 1440-1451.

14 Widdows $\mathrm{H}$, Cordell S: The ethics of biobanking: key issues and controversies. Health Care Anal 2011; 19: 207-219.

15 Ahmad M, Al Gamal E, Othman A, Nasrallah E: Knowledge, attitudes and practices towards cancer prevention and care in Jordan. Report 2011.

16 Hoeyer K, Olofsson BO, Mjörndal T, Lynöe N: Informed consent and biobanks: a population-based study of attitudes towards tissue donation for genetic research. Scand J Public Health 2004; 32: 224-229.

17 Beskow LM, Friedman JY, Hardy NC, Lin L, Weinfurt KP: Simplifying informed consent for biorepositories: stakeholder perspectives. Genet Med. 2010; 12: 567-572.

18 Murphy J, Scott J, Kaufman D, Geller G, LeRoy L, Hudson K: Public expectations for return of results from large-cohort genetic research. Am J Bioeth 2008; 8: $36-43$ 
19 Bookman EB, Langehorne AA, Eckfeldt JH et al: NHLBI Working Group. Reporting genetic results in research studies: summary and recommendations of an NHLB working group. Am J Med Genet A 2006; 140: 1033-1040.

20 Shalowitz DI, Miller FG: Communicating the results of clinical research to participants: attitudes, practices, and future directions. PLoS Med 2008; 5: e91.

21 Forsberg JS, Hansson MG, Eriksson S: Changing perspectives in biobank research: from individual rights to concerns about public health regarding the return of results. Eur J Hum Genet 2009; 17: 1544-1549.

22 Watanabe $\mathrm{M}$, Inoue $\mathrm{Y}$, Chang $\mathrm{C}$ et al: For what am I participating? The need for communication after receiving consent from biobanking project participants: experience in Japan. J Hum Genet 2011; 56: 358-363.

23 Melas PA, Sjöholm LK, Forsner T et al: Examining the public refusal to consent to DNA biobanking: empirical data from a Swedish population-based study. J Med Ethics 2010; 36: 93-98.
24 Eurobarometer 73.1, Biotechnology Report 2010: coordinated by the DirectorateGeneral for Communication 2010.

25 Human Genetics Commission. Public attitudes to human genetic information. People's Panel Quantitative Study Conducted for the Human Genetic Commission. London: Human Genetics Commission, 2001.

26 Falagas ME, Zarkadoulia EA, Samonis G: Arab science in the golden age (750-1258 C.E.) and today. FASEB J 2006; 20: 1581-1586.

27 Asai A, Ohnishi M, Nishigaki E, Sekimoto M, Fukuhara S, Fukui T: Attitudes of the Japanese public and doctors towards use of archived information and samples without informed consent: preliminary findings based on focus group interviews. BMC Med Ethics 2002; 3: E1.

28 Willison DJ, Keshavjee K, Nair K, Goldsmith C, Holbrook AM: Computerization of Medical Practices for the Enhancement of Therapeutic Effectiveness investigators. Patients' consent preferences for research uses of information in electronic medical records: interview and survey data. BMJ 2003; 326: 31

Supplementary Information accompanies this paper on European Journal of Human Genetics website (http://www.nature.com/ejhg) 